\title{
ACTITUDES ANTE LA MUERTE Y SU RELACIÓN CON LAS REDES DE APOYO SOCIAL EN ADULTOS MAYORES DE CAMPECHE, MÉXICO
}

\author{
Gabriela Isabel Pérez-Aranda \\ Facultad de Humanidades \\ Universidad Autónoma de Campeche \\ gaiperez@uacam.mx \\ Daniel Ramírez-Benítez \\ Facultad de Humanidades \\ Universidad Autónoma de Campeche \\ Danielramirezb39@gmail.com \\ Sinuhé Estrada-Carmona \\ Facultad de Humanidades \\ Universidad Autónoma de Campeche \\ sinuhee@hotmail.com
}

Recepción Artículo: 26 marzo 2021 Admisión Evaluación: 26 marzo 2021

Informe Evaluador 1: 27 marzo 2021

Informe Evaluador 2: 29 marzo 2021

Aprobación Publicación: 30 marzo 2021

\section{RESUMEN}

El objetivo fue analizar la relación entre las redes de apoyo social y las actitudes ante la muerte de los adultos mayores de dos poblaciones con diferente densidad demográfica. Se realizó un estudio correlacional, con un diseño no experimental, transversal, con un enfoque cuantitativo. Participaron 400 adultos mayores de 60 a 75 años de dos localidades del Estado de Campeche, Escárcega y San Francisco de Campeche. Se utilizó un tipo de muestreo no probabilístico por conveniencia. Los instrumentos utilizados fueron los siguientes: 1) Escala de Redes de Apoyo Social para Adultos Mayores, que muestra las redes de apoyo social e informal, el tipo de apoyo, el grado de satisfacción. 2) Escala de Ansiedad Ante la Muerte. 3) Escala Revisada de Depresión ante la muerte. 4) Escala de Obsesión ante la muerte. 5) Escala de Miedo a la muerte de Collett-Lester. Los resultados evidenciaron que los adultos mayores con niveles más altos de redes de apoyo social presentan actitudes más favorables ante la muerte, pues están más satisfechos con el apoyo emocional, instrumental, material e informativo que reciben. En conclusión, existe una relación entre las variables analizadas, de tal forma que, a mayor nivel de redes de apoyo social, habrá actitudes más favorables ante la muerte, y viceversa.

Palabras clave: redes de apoyo; actitudes; adultos mayores; vejez; muerte. 


\section{ABSTRACT}

Attitudes towards death and its relationship with social support networks in older adults in Campeche, Mexico. The objective was to analyze the relationship between social support networks and the attitudes towards death of older adults from two populations with different demographic density. A correlational study was carried out, with a non-experimental, cross-sectional design, with a quantitative approach. 400 older adults from 60 to 75 years old participated, from two locations of the state of Campeche, México; Escarcega and San Francisco de Campeche. A non-probability sampling for convenience was used. The instruments used were the following: 1) Scale of social support networks for older adults, showing the social and informal support networks, the type of support, the degree of satisfaction. 2) Scale of Anxiety Before Death. 3) Revised Scale of Depression before death. 4) Scale of obsession with death. 5) Collett-Lester's Fear of Death Scale. The results showed that older adults with higher levels of social support networks, present more favorable attitudes towards death, as they are more satisfied with the emotional, instrumental, material and informational support they received. In conclusion, there is a relationship between the variables analyzed, such that a higher level of social support networks, there will be more favorable attitudes towards death, and vice versa.

Keywords: support networks; attitudes; older adults; old age; death

\section{INTRODUCCIÓN}

La sociedad mexicana que está en vías de desarrollo se enfrentará a un cambio en el crecimiento de la población en edades avanzadas, ya que el envejecimiento tiene un incremento notable. Este hecho se suscitó a mediados de los noventa, y se observa la continuación durante el inicio del siglo XXI. Este reto para la población en México llevará a alcanzar un porcentaje importante de la población adulta en el año 2050 (28 por ciento), que sin duda dejará ver la preparación de la sociedad para atender a esta población (Zúñiga \& Vega, 2004). A través de los cambios de la población en México, y el incremento de los adultos mayores, necesariamente habrá cambios culturales significativos, ya que la vejez pasará por una redefinición, para así lograr la integración de las personas con edades avanzadas en el plano social del país.

La presente investigación tiene como objetivo general analizar la relación entre las redes de apoyo social y las actitudes ante la muerte de los adultos mayores de dos municipios de México, con la hipótesis de que sí existe relación entre las redes de apoyo social y las actitudes ante la muerte de los adultos mayores, teniendo en cuenta que, a mayores niveles de redes de apoyo social, habrá actitudes más favorables ante la muerte en la vejez. Este estudio tiene un impacto significativo en la sociedad actual, ya que los adultos mayores forman parte integral de las poblaciones, generando cultura, redes y beneficios para el entorno, y con los resultados encontrados, se formará una percepción sobre la importancia que tienen estos en el mundo, y las necesidades que estos requieren para su bienestar social.

A raíz del incremento de la población mayor, es necesario examinar las condiciones de vida actuales de los adultos mayores para así generar estrategias que puedan mejorar las condiciones de vida de esta población en un corto plazo. La estructura de la población crece rápidamente, y es necesario contemplar el envejecimiento (Aparicio, 2002).

En un estudio realizado por Medellín, Rivera, López, Kanán \& Rodríguez-Orozco (2012) en Morelia, México, encontraron que el funcionamiento familiar se relaciona principalmente con la red de apoyo familiar y con el sentimiento de falta de apoyo por parte de las redes sociales, es decir, que una mayor percepción de redes de apoyo familiar y menor percepción de sentimiento de falta de apoyo se relacionan con mejor funcionamiento familiar. Asimismo, encontraron que la única red de apoyo social que se relacionó directamente y de manera estadísticamente significativa con el fun- 
cionamiento familiar fue la red de apoyo familiar. Esta investigación muestra que la familia es la que da el mayor soporte a las necesidades de apoyo social, y en la vejez, los adultos mayores requieren este tipo de apoyo para adecuarse al medio y desenvolverse en diferentes ámbitos.

El apoyo social tiene que ver con la satisfacción de las necesidades básicas de una persona, y estas se observan a través de la interacción con los demás, esta interacción se puede dar a través de la afiliación, el afecto proporcionado, el sentido de pertenencia en la comunidad, la creación de la identidad, la seguridad brindada por otros y la aprobación de los pares (Clemente, 2003).

Las redes de apoyo social ayudan a integrar a los adultos mayores en la sociedad, permitiendo así que ejerzan una ciudadanía activa. Los beneficios de las redes de apoyo son diversos, y entre estos se encuentran el enfrentamiento de tensiones, como la adaptación a cambios físicos, mentales o de salud (de Santos, 2009).

Arias (2009) en un estudio realizado en Argentina, evaluó las redes de apoyo social de 105 adultos mayores, y con respecto a las personas que durante la vejez presentan redes escasas, se analizaron los motivos que han generado esta situación, entre los cuales se encuentran: la pérdida de relaciones en la vejez por la muerte de sus propios familiares y amigos, la jubilación, el ingreso a una residencia y las escasas oportunidades de incluir nuevos vínculos a su red.

Una actitud es una respuesta evaluativa relativamente estable, en relación con un objeto, que tiene consecuencias cognitivas, afectivas y probablemente comportamentales (Colell, 2005), y cuando las actitudes se relacionan con la muerte, generan diferentes sensaciones en el ser humano, y muestran ansiedad, depresión, obsesión e incluso miedo, de tal forma que se ha direccionado al estudio de estas actitudes en la vejez. La ansiedad es una reacción emocional producida por la percepción de señales de peligro o amenaza, reales o imaginados a la propia existencia (Tomás-Sábado y Gómez-Benito, 2003). La depresión es un bajo nivel de afecto positivo y un alto nivel de afecto negativo (González, Herrero, Viña, Ibáñez y Peñate, 2004, citado en Agudelo, Buela-Casal y Spielberger, 2007). La obsesión se relaciona con rumiaciones, ideas persistentes o imágenes intrusivas centradas en la idea de la muerte propia 0 de otras personas significativas (Rangel, 2009). El miedo es una emoción más o menos pasajera que aparece cuando se presiente 0 supone un peligro real o aparente (es decir, que "parece y no es») y concreto o inconcreto (vago, impreciso), que se puede sentir individual o colectivamente (Domínguez, 2003). Cuando estas actitudes se presentan en la vejez, existe un bajo nivel de integración por parte de los adultos mayores, creando así una escasa red de apoyo social.

En un estudio realizado por Zapata-López, Delgado-Villamizar \& Cardona-Arango (2015) con 239 adultos mayores del área urbana de Angelópolis, un municipio de Colombia, se encontró que las mujeres tienen una percepción mayor de soledad que los hombres (mujeres-53.8 \%, hombres$30.2 \%$ ). Por otra parte, se encontró que la mayoría de los adultos mayores se encontraba satisfecho con el apoyo de su familia ( $78.1 \%$ en hombres y $68.5 \%$ en mujeres). Asimismo, se reportó que los adultos mayores de ambos sexos están satisfechos en el ámbito social, ya que éstos opinaron que cuentan con sus amigos ( $78.1 \%$ en hombres y $61.5 \%$ en mujeres).

Los adultos mayores que se encuentran viviendo en residencias, tienen condiciones de vida asociados a síntomas depresivos, así como un poco contacto social que los lleva a tener estos síntomas (Estrada, et al. 2015). Estar alejados del contacto social y faltos de redes de apoyo, de protección y carentes de la satisfacción de las necesidades humanas, lleva a presentar síntomas relacionados con la depresión, debido a que no se cuenta con el soporte por parte de una red familiar, institucional o de amigos.

Según Hernández (2001, citado en Estrada, et al. 2015) un sujeto puede deprimirse por diversos factores, entre los cuales se encuentran la viudez, las relaciones sociales, el apoyo familiar y las circunstancias demográficas, pero no sólo pueden causar depresión, sino algún tipo de trastorno. 
En la actualidad el reto para la sociedad es cambiar la forma como se vive la vejez, lo cual necesariamente implica considerar algunos determinantes identificados [...] como la clase social y el género. El objetivo no es alcanzar más años de vida, sino lograr que los años que se vivan sean con calidad y dignidad, independientemente de ser hombre o mujer y de tener cierta posición social (Pelcastre-Villafuerte, Treviño-Siller, González-Vázquez \& Márquez-Serrano, 2011). La posición social o estatus muchas veces está vinculada con la cantidad de personas que vive en un determinado lugar, por los recursos proporcionados y el avance tecnológico del lugar donde se vive, sin embargo, esto no debe ser una limitante para que los adultos mayores en general reciban la atención necesaria para llevar con dignidad su vejez.

En un estudio realizado por Paz (2016) donde investigaba los factores asociados al episodio depresivo en 1482 adultos mayores de Lima Metropolitana y Callao, en su análisis bivariado mostró asociación significativa $(p<0.05)$ de prevalencia anual de episodio depresivo con estar nada, poco o regularmente satisfecho con el aspecto físico en general, el color de la piel, la inteligencia, la condición socioeconómica, el nivel educativo alcanzado, las amistades o relaciones sociales y el lugar de residencia. Datos que se relacionan con tener altos niveles de redes de apoyo social, el estar satisfecho con el apoyo proporcionado por las personas que forman parte del entorno de los adultos mayores.

Asimismo, Rodríguez-Martín, Cárdenas-Rodríguez \& Molerio-Pérez (2012), encontraron en una investigación que las vivencias depresivas son las que tienen mayor efecto en la supresión de pensamientos en adultos mayores, seguidas de las vivencias de ansiedad-miedo.

\section{METODOLOGÍA}

El tipo de estudio fue correlacional, con un diseño no experimental-transversal; con un enfoque cuantitativo.

\section{PARTICIPANTES}

400 adultos mayores de 60 a 75 años de dos localidades de México, una aplicación en la localidad de Escárcega ( $n=200$ ), que cuenta con una baja densidad demográfica (zona rural), y otra en la localidad (zona urbana) de San Francisco de Campeche $(n=200)$, que cuenta con una alta densidad demográfica. Se utilizó un tipo de muestreo no probabilístico por conveniencia, ya que se puede acceder a las personas que acepten participar. Esto debido a que se cuenta con el acceso y proximidad de la población a estudiar (Otzen y Manterola, 2017). Se seleccionó directa e intencionadamente los individuos de la población.

A continuación, se describen las características de la muestra: del total de la población, 59\% fueron mujeres y $41 \%$ hombres. El estado civil de la población en general se encuentra dividido de la siguiente manera: $12 \%$ solteros, $75 \%$ casados, $14 \%$ viudos y $2 \%$ divorciados. La escolaridad presentada se distribuye en: $8 \%$ sin escolaridad, $48 \%$ con nivel primaria, $23 \%$ con secundaria, 10 $\%$ con preparatoria, $6 \%$ con una carrera técnica, $4 \%$ con licenciatura, $1 \%$ con maestría. Del 100 $\%$ de la población, $44 \%$ no presenta enfermedades y $56 \%$ sí tiene alguna enfermedad.

\section{INSTRUMENTOS}

Los instrumentos utilizados fueron tomados del manual "Evaluación en psicogerontología" de Ana Luisa Mónica González-Celis Rangel (2009), mismos que se describen a continuación: La Escala de Redes de Apoyo Social para Adultos Mayores (ERASAM), muestra las redes de apoyo social e informal, el tipo de apoyo, así como el grado de satisfacción de los adultos. Es un cuestionario semiestructurado con tres secciones y examina los tipos de apoyo (emocional, material, instrumental e informativo), así como el nivel de satisfacción de los ámbitos familiar (RIF), extrafamiliar 
(RIE) e institucional (RFI). El ERASAM consta de 54 elementos con una confiabilidad de .899, que nos indica un alto nivel de confianza en el instrumento aplicado, de igual manera, en cada uno de Ios factores se encuentra un nivel de confiabilidad alta, el valor del Alfa de Cronbach de RIF es de .858 , de RIE .843 y de RFI .748 .

Una de las escalas de evaluación de las actitudes ante la muerte que se aplicó es la Escala de Ansiedad Ante la Muerte (DAS) de Templer (1970, en Rangel, 2009), que contiene 15 ítems con formato de respuesta dicotómico (verdadero-falso), de los cuales nueve puntúan verdadero $(1,4,8,9$, $10,11,12,13$ y 14) y seis puntúan falso (2, 3, 5, 6, 7 y 15), así, el rango de posibles puntuaciones totales será de 15 a 30 y las puntuaciones más altas indicarán que existe un mayor nivel de ansiedad ante la muerte. El Alfa de Cronbach de esta escala es de .815.

También se aplicó la Escala Revisada de Depresión ante la muerte (DDS-R) de Tomás-Sábado, Limonero, Templer \& Gómez-Benito (2004-2005, en Rangel, 2009), que consta de 21 ítems, y tiene un formato de respuesta tipo Likert de 5 puntos. Todos los ítems puntúan la misma dirección, de 1 a 5 , donde 1 es igual a total desacuerdo y 5 total acuerdo, con un rango de posibles puntuaciones totales entre 21 y 105, las puntuaciones más altas indicarán que existe un mayor nivel de depresión ante la muerte. El Alfa de Cronbach de esta escala es de .913.

También se aplicó la Escala de Obsesión ante la muerte (DOS) de Tomás-Sábado y GómezBenito (2002-2003, en Rangel, 2009), que contiene 15 ítems con formato de respuesta tipo Likert de 5 puntos. Todos los ítems puntúan con la misma dirección, de 1 a 5 , donde 1 es igual a total desacuerdo y 5 total acuerdo, de manera que el rango de posibles puntuaciones totales fluctuará entre 15, que indicará el nivel mínimo de obsesión ante la muerte, y 75, para el máximo. El Alfa de Cronbach de esta escala es de .904 .

La última escala aplicada es la Escala de Miedo a la muerte del Collett-Lester (CLFDS) de TomásSábado, Limonero y Abdel-Khalek (2007, en Rangel, 2009), que consta de cuatro subescalas: miedo a la propia muerte, miedo al propio proceso de morir, miedo a la muerte de otros y miedo al proceso de morir con otros, con siete ítems para cada una de las subescalas. La escala presenta un tipo de respuesta Likert de 5 puntos en la misma dirección. El Alfa de Cronbach de esta escala es de .941.

Para ubicar el nivel de redes de apoyo social y las actitudes ante la muerte, se utilizaron percentiles, que indican un nivel bajo (0-35), medio (35-70) y alto (70-100).

\section{PROCEDIMIENTO}

Se solicitó la participación de 400 adultos mayores y se les proporcionó una carta de consentimiento informado a cada participante, donde se explicó los objetivos de la investigación. Los participantes que firmaron la carta fueron considerados para la investigación. Se proporcionó a cada participante el ERASAM y las diferentes escalas de las actitudes ante la muerte (ansiedad, depresión, obsesión y miedo). Se registraron las características de cada participante, como edad, sexo, escolaridad, estado civil, presencia de alguna enfermedad, etc. Se pidió a cada participante que respondiera las preguntas de los instrumentos aplicados y se calificaron los instrumentos conforme a las indicaciones del manual.

El trabajo de investigación fue aprobado por la academia de psicología y el comité de ética e investigación de la facultad de humanidades de la Universidad Autónoma de Campeche, México.

\section{ANÁLISIS DE DATOS}

Se registró una base de datos en el software Paquete Estadístico para las Ciencias Sociales (SPSS) versión 23, y se realizaron las siguientes pruebas de hipótesis: $r$ de Pearson para analizar la relación entre las variables y t de Student para comparar grupos independientes y buscar diferencias significativas entre los grupos. Se organizaron los resultados obtenidos en diferentes tablas (inferenciales y correlacionales). 


\section{RESULTADOS}

Tabla 1

Prueba t de Student de las Redes de Apoyo Social de dos localidades

\begin{tabular}{lccccc}
\hline & Localidad & Media & Percentil & Nivel & $\begin{array}{c}\text { Sig. } \\
\text { (bilateral) }\end{array}$ \\
\hline Red Informal Familiar & Urbana & 52.78 & 30 & Bajo & $.000^{*}$ \\
(RIF) & Rural & 64.98 & 65 & Medio & \\
Red Informal Extrafamiliar & Urbana & 31.68 & 40 & Medio & $.000^{*}$ \\
(RIE) & Rural & 39.54 & 60 & Medio & \\
Red Formal Institucional & Urbana & 15.72 & 30 & Bajo & $.000^{*}$ \\
(RFI) & Rural & 20.02 & 75 & Alto & \\
Red Global de Apoyo & Urbana & 100.18 & 25 & Bajo & $.000^{*}$ \\
Social (RGAS) & Rural & 124.54 & 65 & Medio & \\
\hline
\end{tabular}

* Dato estadísticamente significativo $(\mathrm{p}<0.05)$.

A través de la prueba t, se obtuvieron los resultados de la tabla anterior, se muestra la comparación realizada de las redes de apoyo social en dos localidades del estado de Campeche.

El ERASAM está dividido en tres factores y/o tipos diferentes de apoyo y uno global. Se muestran los datos significativos $(p<0.50)$.

En la RIF, las medias muestran una diferencia evidente, y tomando en cuenta los percentiles se observa que la localidad de Escárcega tiene un mayor nivel de apoyo informal familiar. Con respecto a la RIE, se ubica a ambas localidades en el mismo nivel (medio), sin embargo, con base en los percentiles, se observa que, en la localidad de Escárcega, Ios adultos mayores tienen mayor apoyo informal extrafamiliar. En la RFI, se observa una diferencia aún más evidente, y tomando las medias y los percentiles de ambas localidades, se ubica a los adultos mayores de Escárcega (zona rural) en un nivel alto, y a los de Campeche (zona urbana) en un nivel bajo de apoyo formal institucional.La RGAS, que incluye los tres factores antes mencionados, muestra también una notable diferencia entre ambas localidades, y se ubica a los adultos mayores de la localidad de Escárcega en un nivel medio, y a los de Campeche en un nivel bajo de apoyo social.

Esta tabla muestra los resultados por cada factor y/o tipo de apoyo social, y el apoyo global, y así se observa que, en cada uno, los adultos mayores de la localidad de Escárcega tienen mayor nivel de apoyo social frente a los de Campeche. El resultado más notorio, es el mayor nivel de RFI de los adultos mayores de Escárcega con un nivel alto, y el nivel bajo que presentan los de Campeche.

Tabla 2

Prueba $t$ de Student de las actitudes ante la muerte de dos localidades

\begin{tabular}{lccccc}
\hline & Localidad & Media & Percentil & Nivel & $\begin{array}{c}\text { Sig. } \\
\text { (bilateral) }\end{array}$ \\
\hline \multirow{2}{*}{ Ansiedad } & Urbana & 22.18 & 75 & Alto & $.000^{*}$ \\
& Rural & 18.74 & 35 & Bajo & \\
Depresión & Urbana & 43.66 & 80 & Alto & $.000^{*}$ \\
& Rural & 31.54 & 45 & Medio & \\
Obsesión & Urbana & 22.70 & 75 & Alto & .125 \\
& Rural & 19.94 & 50 & Medio & $.000^{*}$ \\
Miedo & Urbana & 78.24 & 80 & Alto & .00 \\
& Rural & 60.76 & 45 & Medio & \\
\hline
\end{tabular}

* Dato estadísticamente significativo $(\mathrm{p}<0.05)$. 
Con base en los resultados obtenidos de la prueba t, en la tabla anterior se observa la comparación de las actitudes ante la muerte de dos localidades. Se muestran los datos significativos $(p<0.05)$.

En la actitud de ansiedad, los adultos mayores de la localidad de Escárcega (zona rural) tienen menor nivel que los de la localidad de Campeche (zona urbana), por lo que se ubica a los adultos mayores de Campeche con un nivel alto de ansiedad, y a los de Escárcega con un nivel bajo. En la actitud de depresión, se observa una diferencia, habiendo mayor depresión en los adultos mayores de Campeche que en los de Escárcega. En la actitud de obsesión, se tiene una sig. Bilateral de .125, lo que indica que no es un dato significativo, sin embargo, tomando en cuenta las medias de ambas localidades, se ubica a los participantes de la localidad de Escárcega con menor obsesión que los de Campeche. Los participantes de Campeche tienen un nivel alto de obsesión, y los de Escárcega un nivel medio. Por otra parte, en la actitud de miedo se observa que los adultos mayores de la localidad de Campeche tienen mayor miedo que los de Escárcega.

Comparando cada una de las actitudes ante la muerte, se observa que, en la localidad de Campeche, se tienen mayores niveles de estas (ansiedad, depresión, obsesión y miedo).

Tabla 3

r de Pearson de la edad, Ios factores de la Red de Apoyo Social, la Red Global de Apoyo Social y las Actitudes ante la muerte

\begin{tabular}{|c|c|c|c|c|c|c|c|}
\hline $\begin{array}{c}\text { Variab } \\
\text { les }\end{array}$ & RIF & RIE & RFI & $\begin{array}{c}\text { Ansieda } \\
\text { d }\end{array}$ & $\begin{array}{c}\text { Depresió } \\
n\end{array}$ & Obsesión & Miedo \\
\hline RIE & $.405^{* *}$ & - & & & & & \\
\hline RFI & $.350^{* *}$ & $.578^{* *}$ & - & & & & \\
\hline $\begin{array}{l}\text { Depre } \\
\text { sión }\end{array}$ & $-.272^{* *}$ & $.334^{* *}$ & $\overline{-}^{*} .311^{* *}$ & $.392^{* *}$ & - & & \\
\hline $\begin{array}{l}\text { Obsesi } \\
\text { ón }\end{array}$ & & $270^{* * *}$ & & $.282^{* *}$ & $.760^{* *}$ & - & \\
\hline Miedo & $-.257^{* *}$ & $.352^{* *}$ & $.281^{* *}$ & $408^{* *}$ & $.715^{* *}$ & $.620^{* *}$ & - \\
\hline RGAS & $852^{* *}$ & $798^{* *}$ & $-670^{* *}$ & & $-.373^{* *}$ & $-.249^{*}$ & $-.366^{* *}$ \\
\hline
\end{tabular}

En la tabla anterior se observa la correlación de la edad, las Redes de Apoyo Social y las actitudes ante la muerte (ansiedad, depresión, obsesión y miedo). Se muestran sólo los datos significativos en 0.01 y 0.05 de la sig. Bilateral.

Se muestra que la edad no se relaciona de manera estadísticamente significativa con ninguna variable, ya sea de las redes de apoyo social o las actitudes ante la muerte, por lo que la edad no tiene relación en tener mayor o menor red de apoyo 0 actitudes ante la muerte.

Observando la correlación de la RIF (Red Informal Familiar) con las diferentes redes de apoyo social y las actitudes ante la muerte, se encuentra lo siguiente: existe una correlación positiva con la RIE, esto quiere decir que, a mayor RIF, habrá mayor RIE. De igual forma, se encuentra una correlación con dirección positiva con RFI, esto indica que a mayor RIF, mayor será la RFI. Ubicando la correlación de la RIF con la actitud de depresión, se ve que hay una correlación con dirección nega- 
tiva, esto es, que a mayor RIF, menor será la actitud depresión, o en su caso, a mayor depresión, menor será la RIF. También, existe una correlación bilateral con la actitud d miedo, que indica lo siguiente: a mayor RIF, menor miedo. Asimismo, se observa una correlación con la RGAS, revelando así que, a mayor RIF, mayor RGAS.

Observando las correlaciones de la RIE (Red Informal Extrafamiliar) con las diferentes redes de apoyo social y las actitudes ante la muerte, se encuentra lo siguiente: existe una correlación con la $\mathrm{RFI}$, que indica que a mayor RIE, mayor será la RFI, y viceversa. Se muestra también una correlación con la actitud de depresión, indicando así que, a mayor RIE, menor será la depresión, o que, a mayor depresión, menor será la RFE. De igual forma, se encuentra un resultado significativo con las actitudes de obsesión y miedo, así como una correlación con dirección negativa, que muestra que a mayor RIE, menores serán las actitudes de obsesión y miedo, y/o que, a mayor obsesión y miedo, menor será la RIE. También, se encuentra una correlación con dirección positiva con la RGAS, que quiere decir: a mayor RIE, mayor RGAS, o viceversa.

Por otra parte, en las correlaciones de la RFI (Red Formal Institucional) con las actitudes ante la muerte, se obtienen correlaciones bidireccionales que muestran lo siguiente: una correlación con dirección negativa con la actitud de depresión, esto significa que a mayor RFI, menor será la depresión. De igual forma, ubicando la correlación con la actitud de miedo se obtiene que a mayor RFI, menor será el miedo de los adultos mayores, y que a mayor miedo, menor será la RFI de estos últimos. Por último, la correlación con la RGAS refleja que a mayor RFI, mayor será la RGAS, y viceversa.

También se muestran las correlaciones de las actitudes ante la muerte con las diferentes redes de apoyo social, las cuales ya fueron descritas anteriormente, sin embargo, se encuentra una significancia entre las propias actitudes ante la muerte. La actitud de ansiedad tiene una correlación con la actitud de depresión que indica: a mayor ansiedad, mayor depresión y viceversa. Asimismo, se observa una correlación con la actitud de obsesión, mostrando así que, a mayor ansiedad, mayor será la obsesión presentada por los adultos mayores.

Otra actitud ante la muerte es la depresión, y ya se han descrito las correlaciones con las redes de apoyo social, sin embargo, se ha encontrado relación con las otras actitudes ante la muerte: se observa que, con la obsesión, a mayor depresión, habrá mayor obsesión ante la muerte, y viceversa. De igual manera, los resultados indican que, a mayor actitud de depresión, habrá menos Red Global de Apoyo Social, y/o que a mayor RGAS, habrá menor depresión. Otra de las actitudes ante la muerte de las que se tiene una correlación es la obsesión, y se muestra que, a mayor obsesión, mayor será el miedo presentado por los adultos mayores y viceversa. De igual manera, con la Red Global de Apoyo Social, Ios resultados indican que, a mayor obsesión, menor será la RGAS, o en su caso, a mayor RGAS, menor obsesión.

Como último punto, se tiene la correlación de la actitud de miedo ante la muerte, con las RGAS, se observa que, a mayor actitud de miedo, menor será la RGAS, y que a mayor RGAS, menor será el miedo presentado por los adultos mayores.

\section{DISCUSIÓN}

Santos (2010, citado en Estrada, et al. 2015) menciona que vivir en residencias hace que las condiciones de vida y el poco contacto social se conviertan en síntomas de depresión, es por esto por lo que, las redes de apoyo social intervienen en la adecuación de los adultos mayores en su contexto social, desde la relación con su familia y amigos. Este dato concuerda con los resultados obtenidos, que muestran que los adultos mayores de la localidad de Escárcega tienen mayores niveles de redes de apoyo social y menores niveles de actitudes ante la muerte, mientras que los adultos mayores de la localidad de Campeche tienen menor nivel de redes de apoyo social y mayores nive- 
les de actitudes ante la muerte, es decir, que los adultos pertenecientes a la localidad de Campeche cuentan con una mejor infraestructura, y viven en mejores condiciones de servicios que los adultos mayores de la localidad de Escárcega, sin embargo, esto los ha llevado a tener menor contacto social y por lo tanto, a presentar síntomas depresivos.

En la presente investigación se encontró una correlación significativa entre los factores de las Redes de Apoyo Social. De la Red Informal Familiar hay una correlación con la Red Informal Extrafamilair, esto quiere decir que, a mayor Red Informal Familiar, habrá mayor Red Informal Extrafamiliar. Los resultados obtenidos concuerdan con un estudio realizado por Zapata-López, et al. (2015), quien encontró que el apoyo de la familia brinda satisfacción a los adultos mayores con un alto porcentaje, asimismo, otras personas como los amigos pertenecientes al ámbito social satisfacen a los adultos mayores de ambos sexos.

Otro de los resultados significativos de este estudio, indica que, a mayor actitud de depresión, habrá menos Red Informal Familiar. Dato que concuerda con Hernández (2001, citado en Estrada, et al. 2015), que menciona que la viudez, las relaciones sociales, el apoyo familiar y circunstancias demográficas, llevan a un sujeto a presentar depresión, o padecer algún tipo de trastorno. Esto muestra que cuando no se tienen buenas redes de apoyo social, que incluyan las relaciones con los familiares, amigos e instituciones, la actitud presentada ante la muerte puede convertirse en depresión. Las circunstancias demográficas, el aumento de la población o la escasez de servicios también aumentan las actitudes ante la muerte de los adultos mayores.

Los resultados de esta investigación muestran que mientras exista una Red Global de Apoyo Social, que incluya los diferentes factores de apoyo (RIF, RIE, RFI), menores serán las actitudes ante la muerte, datos que concuerdan con un estudio realizado por Paz (2016) donde investigaba los factores asociados al episodio depresivo en los adultos mayores, datos que se relacionan con tener altos niveles de redes de apoyo social, el estar satisfecho con el apoyo proporcionado por las personas que forman parte del entorno de los adultos mayores.

Existe una relación entre las redes de apoyo social y las actitudes ante la muerte en los adultos mayores de las dos localidades con diferente densidad demográfica (Escárcega y Campeche), de tal forma que, a mayor nivel de redes de apoyo social, habrá menor nivel de actitudes ante la muerte.

Las redes de apoyo social son mejores en la localidad de Escárcega, población que cuenta con menor densidad demográfica.

Existe un mayor nivel de actitudes de ansiedad, depresión, obsesión y miedo ante la muerte en los participantes de Campeche que en los adultos mayores de la localidad de Escárcega, población con mayor densidad demográfica. La relación principal de cada factor y variable es: a mayores redes de apoyo social, habrá menores actitudes ante la muerte en los adultos mayores de ambas localidades.

Las redes de apoyo social disminuyen las actitudes ante la muerte, es decir, que mientras más proximidad se tenga con los familiares, amigos, parientes, comunidad y las diferentes instituciones, se tendrán mejores condiciones de vida, incluyendo una mejor actitud ante el enfrentamiento propio del proceso de morir, y el proceso de morir de otros; tener redes de apoyo social, ya sea informal o formal, dan como resultado mejor integración en el ámbito social de los adultos mayores.

\section{REFERENCIAS BIBLIOGRÁFICAS}

Agudelo, D., Buela-Casal, G., \& Spielberger, C. (2007). Ansiedad y depresión: el problema de la diferenciación a través de los síntomas. Salud mental, 30(2), 33-41.

Aparicio, R. (2002). Transición demográfica y vulnerabilidad durante la vejez. La situación demográfica de México, 155-167.

Arias, C. (2009). La red de apoyo social en la vejez. Aportes para su evaluación. Revista de 
Psicología da IMED, 1(1), 147-158.

Clemente, M. (2003). Redes sociales de apoyo en relación al proceso de envejecimiento humano. Revisión bibliográfica [The relation between social supports networks and the process of human aging Bibliographical review]. Interdisciplinara, 20(1), 31-60.

Colell, R. (2005). Análisis de las actitudes ante la muerte y el enfermo al final de la vida en estudiantes de enfermería de Andalucía y Cataluña. Universidad Autónoma de Barcelona.

de Santos, Z. (2009). Adulto mayor, redes sociales e integración. Trabajo social (Universidad Nacional de Colombia), (11), 159-174.

Domínguez, V. (2003). El miedo en Aristóteles. Psicothema, 15 (4), 662-666

Estrada, A., Cardona, D., Segura, A., Ordóñez, J., Osorio, J., \& Chavarriaga, L. (2015). Síntomas depresivos en adultos mayores institucionalizados y factores asociados. 12(1), 81-94.

Medellín, M., Rivera, M., López, J., Kanán, M., \& Rodríguez-Orozco, A. (2012). Funcionamiento familiar y su relación con las redes de apoyo social en una muestra de Morelia, México. Salud mental, 35(2), 147-154.

Otzen, T., \& Manterola, C. (2017). Técnicas de Muestreo sobre una Población a Estudio. International Journal of Morphology, 35(1), 227-232.

Paz, V. (2016). Factores Asociados al Episodio Depresivo en los Adultos Mayores de Lima Metropolitana y Callao. Revista ANALES DE SALUD MENTAL, 31(1).

Pelcastre-Villafuerte, B., Treviño-Siller, S., González-Vázquez, T., \& Márquez-Serrano, M. (2011). Apoyo social y condiciones de vida de adultos mayores que viven en la pobreza urbana en México. Cadernos de Saúde Pública, 27, 460-470.

Rangel, A. (2009). Evaluación en psicogerontología. Editorial El Manual Moderno.

Rodríguez-Martín, B. C., Cárdenas-Rodríguez, R., \& Molerio-Pérez, 0. (2012). Predictores de la supresión de pensamientos en jóvenes y adultos mayores: preocupación y vivencias. Pensando Psicología, 8(14), 19-27.

Tomás-Sábado, J., \& Gómez-Benito, J. (2003). Variables relacionadas con la ansiedad ante la muerte. Revista de psicología general y aplicada: Revista de la Federación Española de Asociaciones de Psicología, 56(3), 257-279.

Zapata-López, B., Delgado-Villamizar, N., \& Cardona-Arango, D. (2015). Apoyo social y familiar al adulto mayor del área urbana en Angelópolis. Colombia 2011. Revista de Salud Pública, 17, 848860.

Zúñiga, E., \& Vega, D. (2004). Envejecimiento de la población de México, reto del siglo XXI. México: Consejo Nacional de Población. 